\title{
TITLE: INFORMATION THEORETIC DERIVATIUN OF NETWORK ARCHITECTURE AND LEARNING ALGORITHMS
}

AUthoR(S). R. D. Jones, C. W. Barnes, Y. C. Lee, W. C. Mead

\author{
SUBMITTED TO: IJCNN-9l-Seattle Conference \\ Seattle, WA
}

\section{DISCLAIMER}

This report was prepared as an account of work sponsored by an agency of the United States Government. Neither the United States Government nor any agency thereof, nor any of their employees, makes any warraniy, express or implied, or assumes any legal liability or responsibility for the accuracy, completeness, or usefulness of any information, apparatus, product, or process disclosed, or represents that its use would not infringe privately owned rights. Reference herein to any specific commercial product, process, or service by trade name, trademark, manufacturer, or otherwise does not necessarily constitute or imply its endorsement, recommendation, or favoring by the United States Government or any agency thereof. The views and opinions of authors expressed herein do not necessarily state or reflect those of the United States Government or any agency thereof. 


\title{
INFORMATION THEORETIC DERIVATION \\ OF NETWORK ARCHITECTURE AND LEARNING ALGORITHMS
}

\author{
R. D. JONES ${ }^{a}$, C. W. BARNES, Y. C. LEE, and W. C. MEAD
}

Los Alamos National Laboratory

Los Alamos, New Mezico 87545 USA

ardjOlanl.gov

\begin{abstract}
Using variational techniques, we derive a feedforward network architecture that minimizes a least squares cost function with the soft constraint that the mutual information between input and output be maximized. This permits optimum generalization for a given accuracy. A set of leearning algorithms are also obtained. The network and learning algorithms are tested on a set of test problems which emphasire time series prediction.
\end{abstract}

\section{Introduction}

The goal of feedforward networks is to maximize network approximation accuracy while also maximizing network generalizability. The accuracy of the output is typically obtained by implementation of learning algorithms which minimize a least squares cost function. Generalizability can be obtained through maximization of a cost function proportional to the mutual information between input and output. ${ }^{1}$ These techniques generalize work of Kohonen. ${ }^{2}$ In this paper we demonstrate how the optimization of a composite cost function that contains both a least squares term and a mutual information term can lead to an architecture and to learning algorithms which yield optimum generalization for a given accuracy.

\section{The Architecture}

We can now examine the probability distribution which minimizes the cost function

$$
F \equiv E-\tau S
$$

where

$$
E=\frac{N}{2} \int_{I} d x \int_{O} d y \epsilon(x, y) P(y \mid x) P(x)
$$

and

$$
S=N \int_{I} d x \int_{O} d y P(y \mid x) P(x) \log \left[\frac{P(y \mid x)}{P(y)}\right] .
$$

Here, $c(x, y)$ is

$$
\epsilon(x, y) \equiv \frac{1}{2}[y-\phi(x)]^{T}[y-\phi(x)]
$$

where

$$
\phi(x)=\int_{O} d y y P(y \mid x)
$$

and

$$
P(y)=\int d x P(y \mid x) P(x) .
$$

Here, $E$ is the least squares portion of the cost function and $S$ is the mutual information. We find the functional form of $P(y \mid x)$ by equating the functional deriative to zero. We obtain a relation between 
the conditional probability, $P(y \mid x)$ known as the posterior, and the pre-measurement probability, $P(y)$ known as the prior. In general, these two probabilities are related through Bayes theorem,

$$
P(y \mid x)=\frac{P(x \mid y)}{P(x)} P(y)
$$

where

$$
P(x)=\int_{0} d y P(x \mid y) P(y) .
$$

The conditional probability, $P(x \mid y)$, is known as the likelihood.

We can regard Eqs. 2 and 3 as well as the normalization condition,

$$
1=\int_{0} d y P(y \mid x)
$$

as soft constraints. The cost function can be modified to read

$$
F^{\prime}=N \int_{I} d x \int_{O} d y P(y \mid x) P(x)\left[\epsilon(x, y)-\tau \log \left[\frac{P(y \mid x)}{P(y)}\right]+\alpha(x)+\beta(y)+\gamma^{T}(x) y\right]
$$

where $\alpha(x) \in \Re, \beta(y) \in R e$, and $\gamma(x) \in \Re^{m}$ are functions that are determined by the constraints. Setting the functional derivative,

$$
\frac{\delta F^{\prime}}{\delta P(y \mid x)}
$$

equal to zero yields

$$
\epsilon(x, y)-\tau \log \left[\frac{P(y \mid x)}{P(y)}\right]+\alpha(x)+\beta(y)+y \gamma(x)-\tau=0 .
$$

Solving for $P(y \mid x)$ obtains

$$
\phi(x)=\frac{1}{P(x)} \int_{O} d y y \frac{E(x, y)}{\int d x E(x, y)} P(y)
$$

where

$$
E(x, y) \equiv \exp \left[-\frac{y^{T} y-(\gamma(x)-\phi(x))^{T} y+\phi^{T}(x) \phi(x)}{2 \tau}\right] .
$$

This is an integral equation which contains the arbitrary function, $\gamma(x)$. In general, $\gamma(x)$ cannot be chosen such that Eq. 3 is exactly satisfied. However, it can be approximately satisfied if

$$
\gamma(x) \equiv 2 \phi(x) \text {. }
$$

This forces the integrand in Eq. 3 to be sharply peaked around $y=\phi(x) . E(x, y)$ becomes

$$
E(x, y)=\exp \left[-\frac{\epsilon(x, y)}{\tau}\right]
$$

We see from Eq. 2 that the posterior is

$$
P(y \mid x)=\frac{1}{P(x)} \frac{E(x, y)}{\int d x E(x, y)} P(y) .
$$

This yields for the likelihood

$$
P(x \mid y)=\frac{E(x, y)}{\int d x E(x, y)}
$$




\section{R. D. Jones et al. / Information Theoretic Derivation...}

The output of the network can be written

$$
\phi(x)=\frac{1}{P(x)} \int_{O} d y \text { y } P(x \mid y) P(y) .
$$

Equations 3 to 6 define a near optimum architecture given the soft constraints imposed on the least squares minimization. From Eqs. 5 and 4 it can be seen that the role of the Lagrange multiplier, $\tau$, is to spread the input probability, $P(x)$. This will degrade the fit of $\phi(x)$ to $f(x)$ but it will improve the generalizability of the network. For very large values of $\tau$ the network only acquires the average value of $f(x)$. This is the ultimate generalization.

Suppose we have just presented the network with $N$ training pairs and we had exact knowledge of the desired output. Then $P(y)$ is given by

$$
P(y)=\frac{1}{N} \sum_{p=1}^{N} \delta\left(y-f\left(x_{p}\right)\right) .
$$

Thus $P(x)$ can be written

$$
P(x)=\int d y P(x \mid y) P(y)=\frac{1}{N} \sum_{p=1}^{N} P\left(x \mid f\left(x_{p}\right)\right) .
$$

The posterior becomes

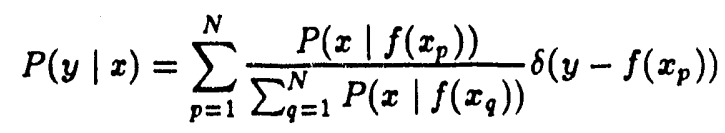

and the network output becomes

$$
\phi(x)=\frac{\sum_{p=1}^{N} f\left(x_{p}\right) P\left(x \mid f\left(x_{p}\right)\right)}{\sum_{q=1}^{N} P\left(x \mid f\left(x_{q}\right)\right)} .
$$

This architecture is a linear superposition of nonlinear elements. We can make the identifications

$$
\begin{gathered}
P(y \mid x)=\sum_{j=1}^{M} u\left(x ; b_{j}\right) v\left(y-a_{j}\right) \\
u\left(x ; b_{p}\right) \equiv P(p \mid x)=\frac{P\left(x \mid f\left(x_{p}\right)\right)}{\sum_{q=1}^{N}} \frac{P\left(x \mid f\left(x_{q}\right)\right)}{}
\end{gathered}
$$

and

$$
v\left(y-f\left(x_{p}\right)\right) \equiv P(y \mid p)=\delta\left(y-f\left(x_{p}\right)\right) .
$$

Moreover, we can write the specific form of the nonlinear element as

$$
u\left(x, b_{p}\right)=\left[\sum_{q=1}^{N} \frac{E\left(x, f\left(x_{q}\right)\right)}{\int d x E\left(x, f\left(x_{q}\right)\right)}\right]^{-1} \frac{E\left(x, f\left(x_{p}\right)\right)}{\int d x E\left(x, f\left(x_{p}\right)\right)},
$$

where

$$
E\left(x, f\left(x_{p}\right)=\exp \left[-\frac{\epsilon\left(x, f\left(x_{p}\right)\right)}{\tau}\right] .\right.
$$

Equations 7 and 8 give the optimum architecture given that the desired output is perfectly known. The architecture is self-consistant. The output must be known in order to specify $\epsilon$ and $\epsilon$ must be known 


\section{R. D. Jones et al. / Information Theoretic Derivation...}

in order to specify the output. We can remove the self-consistancy in the limit of small $\tau$. In that case the function $E\left(x, f\left(x_{p}\right)\right)$ is sharply peaked about $\phi(x)=f\left(x_{p}\right)$. If we Taylor expand $\phi(x)$ about $f\left(x_{p}\right)$ the energy, $\epsilon$, becomes

$$
\epsilon\left(x, f\left(x_{p}\right)\right) \approx \frac{1}{2}\left[\left(x-x_{p}\right)^{T}\left(\frac{\partial \phi(x)}{\partial x}\right)_{x=x}\right]\left[\left(x-x_{p}\right)^{T}\left(\frac{\partial \phi(x)}{\partial x}\right)_{x=x_{p}}\right]^{T} .
$$

Here,

$$
\left(\frac{\partial \phi(x)}{\partial x}\right)_{x=x_{p}}
$$

is an $n \times m$ matrix. Equation 7 becomes

$$
u\left(x, b_{p}\right)=\frac{\left|\gamma_{p}^{-1}\right|^{-1 / 2} \exp \left[-\left(x-x_{p}\right)^{T} \gamma_{p}\left(x-x_{p}\right)\right]}{\sum_{q=1}^{N}\left|\gamma_{q}^{-1}\right|^{-1 / 2} \exp \left[-\left(x-x_{q}\right)^{T} \gamma_{q}\left(x-x_{q}\right)\right]}
$$

where $\gamma_{p}$ is an $n \times n$ matrix given by

$$
\gamma_{p} \equiv \frac{1}{2 \tau}\left(\frac{\partial \phi(x)}{\partial x}\right)_{x=x_{p}}\left(\frac{\partial \phi(x)}{\partial x}\right)_{x=x_{p}}^{T} .
$$

If the data is preprocessed such that each input affects the output nearly independently and redundant. and irrelevant inputs are not present then Eq. 9 can be simplified to

$$
u\left(x, b_{p}\right)=\frac{\beta_{p}^{n / 2} \exp \left[-\beta_{p}\left(x-x_{p}\right)^{T}\left(x-x_{p}\right)\right]}{\sum_{q=1}^{N} \beta_{q}^{n / 2} \exp \left[-\beta_{q}\left(x-x_{q}\right)^{T}\left(x-x_{q}\right)\right]}
$$

where $\gamma$ can be written as a scalar $\beta$ times a unit matrix. Writing Eq. 9 as Eq. 10 is a serious and drastic approximation. The process of simplifying the matrix $\gamma_{p}$ is known as feature extraction.

The network output becomes

$$
\phi(x)=\sum_{p=1}^{N} f\left(x_{p}\right) u\left(x, b_{p}\right)
$$

where $u$ is given by Eq. 10. We will permit the quantities $f\left(x_{p}\right), x_{p}$, and $\beta_{p}$ to be adjustable parameters that minimize a given cost function. We can thus associate $\beta_{p}$ and $x_{p}$ with the parameters $b_{p}$ in the function $u\left(x, b_{p}\right)$.

We can relate Eq. 11 to a network architecture. Note the identity,

$$
g(x)=\frac{\sum_{j=1}^{N} g(x) \rho_{j}(x)}{\sum_{j=1}^{N} \rho_{j}(x)}
$$

Here, $\rho_{j}(x)$ is a localized function of $x$ about some $a_{j}$. Hence, $g(x)$ on the right of Eq. 12 can be approximated by its Taylor expansion about $a_{j}$. We have then,

$$
\phi(x)=\sum_{j=1}^{N}\left[f_{j}+\left(x-a_{j}\right) \cdot d_{j}\right] \frac{\rho_{j}(x)}{\sum_{j} \rho_{j}(x)}
$$

for an approximation to $g(x)$. If we compare with Eq. 10 and 11 we can write a specific form for the local function, $\rho$,

$$
\rho_{j}(x)=\beta_{p}^{n / 2} \exp \left[-\beta_{p}\left(x-x_{p}\right)^{T}\left(x-x_{p}\right)\right] .
$$

This net is similar to the radial basis function net of Moody and Darken ${ }^{3}$ but differs in two ways, the use of normalization and also a linear term, $\left(x-a_{j}\right) \cdot d_{j}$. The use of a normalization term was suggested but not pursued by Moody and Darken. The addition of these two terms is responsible for the reduction in the amount of training data needed to obtain reasonable approximations. As in the case with radial basis functions, the training of $f_{j}$ and $d_{j}$ is linear and hence very fast. The widths of the basis functions can also be trained. This training is nonlinear. 


\section{s. Learning Algorithms}

The training for the linear quantities, $f_{j}$ and $d_{j}$ is given by

$$
f_{j}^{p+1}=f_{j}^{p}+\alpha\left[g\left(x_{p}\right)-\phi\left(x_{p}\right)\right] \frac{\rho_{j}\left(x_{p}\right) \sum_{k} \rho_{k}\left(x_{p}\right)}{\sum_{k}\left[\rho_{k}^{2}\left(x_{p}\right)+\beta_{k}\left(x-a_{k}\right)^{2} \rho_{k}^{2}\left(x_{p}\right)\right]}
$$

and

$$
d_{j}^{p+1}=d_{j}^{p}+\alpha\left[g\left(x_{p}\right)-\phi\left(x_{p}\right)\right] \frac{\beta_{j}\left(x_{p}-a_{j}\right) \rho_{j}\left(x_{p}\right) \sum_{k} \rho_{k}\left(x_{p}\right)}{\sum_{k}\left[\rho_{k}^{2}\left(x_{p}\right)+\beta_{k}\left(x-a_{k}\right)^{2} \rho_{k}^{2}\left(x_{p}\right)\right]} .
$$

Here, $x_{p}$ is a training input vector and $g\left(x_{p}\right)$ is the training output for the $p^{\text {th }}$ on-fine presentation of data to the network. The superscripts $p$ and $p+1$ indicate values of the weights before and after the $p^{\text {th }}$ presentation of training data. The learning rate, $\alpha$, is usually taken to be $1 / 3$. This training algorithm or modifications of it have been used in time series prediction and in control. ${ }^{4-6}$

We can also train the basis function widths. Equation 7 can be written

$$
F^{\prime}=N \int_{I} d x \int_{O} d y P(y \mid x) P(x)\left[-\epsilon(x, y)-\tau \log \left[\frac{P(y \mid x) P(x) \int d x E(x, y)}{P(y)}\right]+\tau\right]
$$

We have

$$
\int d x E\left(x, a_{j}\right) \approx\left(\frac{2 \pi}{\beta_{j}}\right)^{n / 2} .
$$

If we apply gradient descent learning to this cost function we find

$$
\begin{gathered}
\beta_{j}^{p+1}=\beta_{j}^{p}-\eta \frac{n \beta_{j}^{p}}{2} \rho\left(x_{p} ; b_{j}^{p}, \beta_{j}^{p}\right) v\left[\phi\left(x_{p}\right)-a_{j}\right] \\
+\eta\left(\beta_{j}^{p}\right)^{2}\left[\frac{n}{2 \beta_{j}^{p}}-\left(x_{p}-b_{j}^{p}\right)^{T}\left(x_{p}-b_{j}^{p}\right)\right] \rho\left(x_{p} ; b_{j}^{p}, \beta_{j}^{p}\right) v\left[\phi\left(x_{p}\right)-a_{j}\right] K\left[x_{p}, \phi\left(x_{p}\right) ; a, b, \beta\right]
\end{gathered}
$$

where $K$ is now given by

$$
K\left(x_{p}, y ; a, b, \beta\right) \equiv \log \left[\frac{\pi\left(x_{p}, y ; a, b, \beta\right) \pi(x ; b, \beta)}{\pi(y ; a) \beta^{n / 2}}\right]+\frac{1}{2 \tau}\left[a_{j}-\phi(x)\right]^{T}\left[a_{j}-\phi(x)\right] .
$$

In each of these algorithms the conscience is affected indirectly by the errors that are being made in the approximation of $f$.

The behavior of this algorithm on the logistic map is displayed in Fig. 1.

\section{Summary}

We have derived a network architecture from first principles. The network is designed to optimize both accuracy and generalization. The architecture resembles local radial basis function networks with two important modifications, a normalization which greatly reduces the data requirements and an extra set of gradient style weights which improves inter;olation. Performing gradient descent on the composite cost function obtains a learning algorithm for the basis function widths which adjusts the widths for good generalization. 

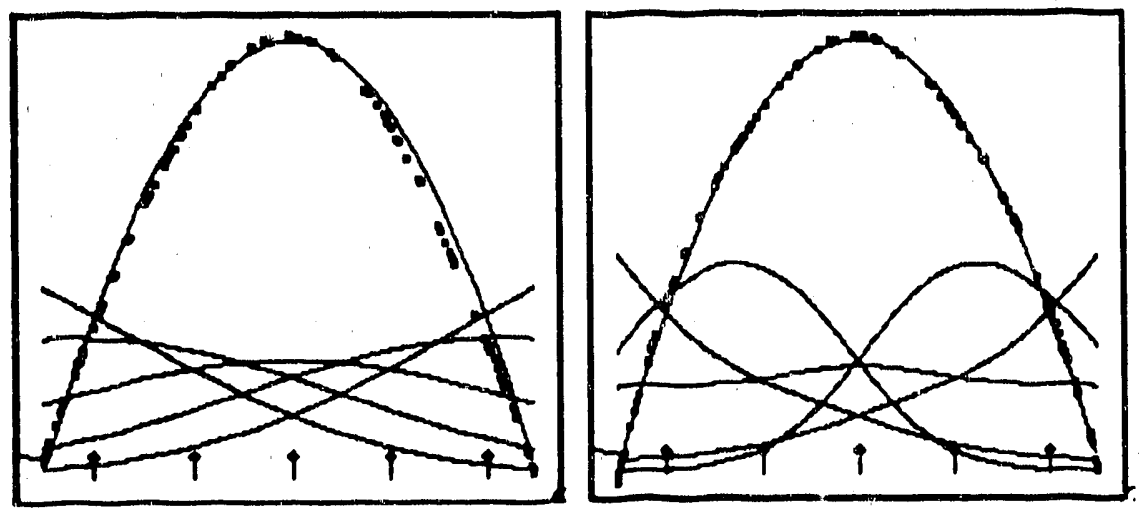

Fig. 1 Behavior of Eq. 1.5-15 on the appraximation of the logistic map. The left plot is with no training of the widths, $\beta_{j}$, and the right plot is with training using Eq. 1.5-3. The upper plot is of $x_{p+1}=4 x_{p}\left(1-x_{p}\right)$ where $x_{p}$ is plotted on the abscissa and $x_{p+1}$ is plotted on the ordinate. The solid line is the desired curve. The dots are the predictions of the network. The training on the gradients, $d$, ws turned off. 20000 training patterns were used. Five evenly spaced basis functions were used. The lociations are indicated by hash marks with diamonds. Also displayed in each plot are the five curves for the five basis functions. Not the shape adjustment in the right plot. The learning rate, $\eta$, was set to 0.3 for the training of the coefficients $f_{j}$ and to 0.03 for the $\beta$ training. The root mean square error normalized to the atandard deviation of the data was 0.075 for the case with no $\beta$ training and 0.035 for the case in which the betas were trained.

\section{References}

1. R. Linsker, "How to generate ordered maps by maximizing the mutual information between input and output signals," Neural Compuration, 1, (1989) 402.

2. T. Kohonen, Self-Organization and Associative Memory, (Springer-Verlag, Berlin, 1989).

3. J. Moody and C. J. Darken, "Fast learning in networks of locally tuned processing units," Neural Computation, 1, (1989) 281-294.

1. R. D. Jones, Y. C. Lee, C. W. Barnes, G. W. Flake, K. Lee, P. S. Lewis and S. Qian, "Function approximation and time series prediction with neural networks," Talk presented at the Center for Nonlinear Studies, Los Alamos National Laboratory, Los Alamos, New Mexico on September 87 , 1989. Los Alamos Report LA.UR-90-21. and in Proceedings of the International Joint Conference on Neural Networks, San Diego, California, June 17-21, 1990. (1990) 1-649-666.

5. W. C. Mead, R. D. Jones, Y. C. Lee, C. W. Barnes, G. W. Flake, L. A. Lee, and M. K. O'Rourke, "Using CNLS-Net to Predict the Mackey-Glass Chaotic Time Series," Proceedings of the International Joint Conference on Neural Networks, Seattle, Washington, 1991.

6. C. W. Barnes, S. K. Brown, G. W. Flake, R. D. Jones, M. K. O'Rourke, and Y. C. Lee "Applications of Neural Networks to Process Control and Modeling," Proceedings of the International Joint Conference on Neural Networks, Seattle, Washington, 1991. 

